\title{
An Energy Saving Strategy for LTE-A Multiantenna Systems
}

\section{Reema Imran •}

Mutaz Shukair •

Nizar Zorba .

\section{Christos Verikoukis}

Received: date / Accepted: date

\begin{abstract}
Long Term Evolution - Advanced (LTE-A) supports closed-loop Multiple Input Multiple Output (MIMO) techniques to improve its performance, but in order to exploit multiuser (MU) MIMO channel capabilities, the design of an efficient Medium Access Control (MAC) scheme that supports MU-MIMO is still an open issue in the literature. This paper proposes a novel energy efficient MAC scheme for LTE-A, which aims to achieve simultaneous downlink transmissions to multiple users, through the deployment of a low complexity beamforming technique at the physical layer. Our proposed scheme takes advantage from the multiuser gain of the MIMO channel and the multiplexing gain of the Multibeam Opportunistic Beamforming (MOB) technique, to improve the system throughput and the energy efficiency of wireless networks. We provide a closed form mathematical expression of the energy efficiency, where the results from the proposed scheme achieve a considerable energy saving at the Base Station (eNB).
\end{abstract}

Keywords Energy Saving · MAC designs · LTE-A · Multiuser MIMO

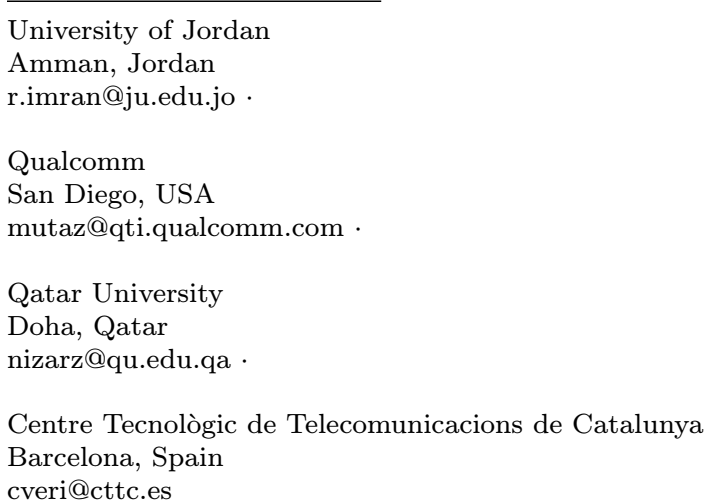




\section{Introduction}

Long Term Evolution - Advanced (LTE-A) [1] is a promising technology that has been recently standardized with the aim to increase the performance of mobile broadband systems. The main targets of this technology are to improve the throughput and the Quality of Service (QoS) [2], among others, while reducing the user and operator costs by employing all the cutting edge techniques like MIMO [3], Orthogonal Frequency Division Multiplexing (OFDM) [4], etc.

LTE-A supports MIMO antenna technology, where multiple antennas are used at the transmitter or/and at the receiver to achieve the ambitious requirements for spectral efficiency, cell coverage, and throughput without additional increase in the bandwidth or transmitted power [5]. The integration of MIMO within LTE-A supports the incorporation of transmission schemes such as transmit diversity, spatial multiplexing and beamforming [6]. Although several MIMO schemes are provisioned in the LTE-A standard, the exact technique to be applied in each case has been left open in the standard [7], so that each manufacturer may decide on the most suitable option for its scenario/application [8].

The most attractive Downlink options of MIMO need for full Channel State Information at the Transmitter (CSIT) before the transmission starts, but its impractical due to the feedback load in the Uplink [9]. This problem is more evident in the Multiuser (MU) scenarios (e.g., cellular systems), as the CSIT from each user is unaffordable [10]. The application of non-CSIT MIMO schemes in Multiuser scenarios does not provide the required boost in data rate and throughput, so that partial-CSIT MIMO schemes will be the most implemented ones. The Multibeam Opportunistic Beamforming (MOB) is one of the highest performance and low complexity schemes in literature [11]. It exploits the multiuser availability and it can offer service to several users at the same time, frequency and code through the transmission over several beams.

Although LTE-A improves the throughput and the end-users' QoS, but the increasing cost of power and the large amount of consumed energy by the base station (named eNB in the LTE-A specifications) has pushed designers to care also about the power/energy efficiency of the eNBs [12]. Developing energy saving techniques in wireless networks is a challenging issue as reducing the transmitted energy, without compromising the QoS, at the eNB reduces the communication costs and minimizes the interference at the cell edges [13] [14].

Energy efficiency can be achieved in wireless networks by switching off different components of the eNB [15], reducing the load of the network [16] and increasing the coverage (range) of the eNB through the effective use of MIMO [12]. Discontinuous transmission (DTX) is supported by LTE-A to allow energy saving at the User Equipment (UE) by turning off its radio circuitry, thus increasing the UEs' battery lifetime [17]. At every predefined DTX cycle time, the UE wakes up and monitors the paging messages and control signaling for a predefined period, if no scheduling assignment is received, the UE sleeps again. Recent studies show that despite the deployment of DTX, LTE-A is 
less energy efficient compared with WiFi and 3G [17], so that energy efficient solutions are required for LTE-A.

In this paper, we propose an energy efficient Medium Access Control (MAC) scheme for multiuser transmission, that utilizes the multiplexing gain and MIMO beamforming at the physical layer to develop energy efficient wireless networks. As the Transmission Mode (TM-5) in the LTE-A specifications is devoted for Multiuser MIMO [18], we will adapt it to our objectives, being backward compatible with the LTE-A standard. We should stress on the point that the predefined throughput will be achieved, while less energy is consumed.

We mathematically formulate the energy consumption for LTE-A based systems. The closed form expressions show the impact of each parameter in the system performance, enabling to calculate the amount of saved energy for our proposal. The results from equations are compared to computer simulations for an LTE-A scenario, where we see a very close behaviour between the mathematical equations and the simulations.

The remainder of this paper is organized as follows: section II deals with the system model and the beamforming transmission technique at the physical layer. A description of the proposed power/energy scheme is shown in section III followed by its inclusion in the LTE-A standard through a Multiuser MAC design in section IV. The simulations and numerical results are shown in section V. The paper ends with the conclusions in section VI.

\section{System Model}

We focus on the single-cell LTE-A downlink channel, where $Q$ physical resource blocks $R B s$ are available with zero interference among them. OFDM guarantees no interference among the RBs. A multiple antenna downlink scenario is considered where the eNB transmitter is equipped with $n_{t}$ transmit antennas to serve $K$ users, where it is assumed that $K \geq n_{t}$. To achieve lower complexity and implementation cost at the receiver side, we assume a single antenna at each UE. Let $\mathbf{x}(t)$ be the $n_{t} \times 1$ transmitted vector, then the $k^{t h}$ user received signal $y_{k}(t)$ is given by

$$
y_{k}(t)=\mathbf{h}_{k}(t) \mathbf{x}(t)+z_{k}(t)
$$

where $z_{k}(t)$ is an additive independent and identically distributed (i.i.d.) complex noise component with zero mean and variance of $E\left\{\left|z_{k}\right|^{2}\right\}=\sigma^{2}$. A channel $\mathbf{h}_{1 \times n_{t}}$ between each of the users and the eNB is considered, following the specular model [19] for flat fading outdoor channel, which is assumed to keep constant through the coherence time $T_{c}$ and independently changes between consecutive time intervals. A total transmit power constraint of $P_{t}=1$ is considered and for ease of notation, time index is dropped whenever possible.

The channel between the $k^{\text {th }}$ user and the eNB is defined as

$$
\mathbf{h}_{k}=\frac{1}{\sqrt{W}} \sum_{w=1}^{W} \alpha_{k, w} \mathbf{a}\left(\theta_{k, w}\right)
$$


where $W$ is the number of paths that the signal is assumed to follow from the eNB to the user $k . \theta_{k, w}$ is the angle of incidence of these paths, which are assumed to have gaussian distribution with mean $\overline{\theta_{k}}$ and angle spread of $A S=\sqrt{E\left(\left|\theta_{k, w}-\overline{\theta_{k}}\right|^{2}\right)}$. On the other hand, $\alpha_{k, w}$ is the gain of the $w^{t h}$ path seen by the $k^{t h}$ user, which is a zero mean complex gaussian distributed random variable; and $\mathbf{a}\left(\theta_{k, w}\right)$ is the steering vector defined as:

$$
\mathbf{a}\left(\theta_{k, w}\right)=\left[1, \exp ^{-j 2 \pi \frac{d \cos \left(\theta_{k, w}\right)}{\lambda}}, . ., \exp ^{-j 2 \pi \frac{\left(n_{t}-1\right) d \cos \left(\theta_{k, w}\right)}{\lambda}}\right]
$$

where $d$ is the distance between the antennas at the eNB and $\lambda$ is the wavelength.

The transmitter delivers service to a maximum of $n_{t}$ simultaneous users in each $R B$. Thus the transmitted signal $\mathbf{x}$ encloses the uncorrelated data symbols $s_{k}$ to each one of the selected users with $E\left\{\left|s_{k}\right|^{2}\right\}=1$.

\subsection{Multibeam Opportunistic Beamforming (MOB)}

One of the major low complexity transmission techniques in multiuser MIMO scenarios is the MOB technique [20], which aims to improve the system performance by enabling the transmitter at the eNB to serve several users at the same time, through generating several beams at the eNB. In order to maintain the lowest interference between the served users, the generated beams have to be orthogonal which restricts the eNB transmitter to generate a maximum of $n_{t}$ beams, and disabling any user to obtain more than one beam at a time [10].

The procedure is that - at the beginning of the transmission process - a reference predefined training sequence is transmitted from the eNB over each one of the available beams; each user in the system calculates its received Signal to Noise and Interference Ratio (SNIR) value corresponding to each beam, and feeds back the maximum measured SNIR value with an integer for the corresponding beam index. The eNB selects the user with the largest SNIR for each one of the beams (i.e., opportunistic scheduling), and the data flow starts.

This multibeam strategy extracts the spatial multiplexing gain by serving several users at the same time achieving high system sum rate. If $n_{t}$ users are selected for transmission, the transmitted signal that encloses the data symbols can be expressed as

$$
\mathbf{x}=\frac{1}{\sqrt{n_{t}}} \sum_{m=1}^{n_{t}} \mathbf{b}_{m} s_{m}
$$

where $s_{m}$ is the data symbol for the $m^{\text {th }}$ selected user and $\mathbf{b}_{m}$ is the normalized transmission beam, so that the term $\frac{1}{\sqrt{n_{t}}}$ makes the transmitted power to be unity. 
This technique has a major drawback that each generated beam causes an interference on non-intended users ${ }^{1}$. The received SNIR value for the $k^{\text {th }}$ user that is served by the $m_{t h}$ beam can be formulated as

$$
\gamma_{k, m}=\frac{\frac{1}{n_{t}}\left|\mathbf{h}_{k} \mathbf{b}_{m}\right|^{2}}{\sigma^{2}+\sum_{v \neq m}^{n_{t}} \frac{1}{n_{t}}\left|\mathbf{h}_{k} \mathbf{b}_{v}\right|^{2}}
$$

where a uniform power allocation among all the users is considered. The $\mathrm{Cu}$ mulative Density Function (CDF) for the serving SNIR is formulated as [10]

$$
F(\gamma)=\left[1-\frac{\exp \left(-n_{t} \gamma \sigma^{2}\right)}{(1+\gamma)^{n_{t}-1}}\right]^{K}
$$

Within this scheme there exists the possibility that no user selects any of the $m^{t h}$ beam or all the SNIR values for that beam are below the minimum threshold for correct detection at the receiver side. In that case, the $m^{\text {th }}$ beam is switched off and its transmit energy is saved.

\section{Energy Saving Scheme}

We consider a Partial CSIT LTE-A scenario with Adaptive Modulation and Coding (AMC) transmission technique [13]. Through the AMC scheme, the transmitter tracks the changes and fluctuations of a wireless channel, and adapts its employed modulation and code to the channel characteristics, where the employed modulation has to be carefully selected to meet a predefined Symbol Error Rate (SER) value. If we assume that $R$ different modulation and coding schemes are available, then the instantaneous SNIR values are divided into $R$ regions with thresholds defined as $\left[\gamma_{1}, ., \gamma_{R}\right]$, so if the measured SNIR value lies within the $r^{t h}$ region $\left(\gamma_{r} \leq S N I R<\gamma_{r+1}\right)$ only the modulation and coding scheme corresponding to that interval can be employed. Using the CDF given in Eqn.(6), the probability that a user lies within the $r^{\text {th }}$ region can be obtained as

$$
\Omega(r)=\int_{\gamma_{r}}^{\gamma_{r+1}} f(\gamma) d \gamma=F\left(\gamma_{r+1}\right)-F\left(\gamma_{r}\right)
$$

where $f(\gamma)$ is the PDF from Eqn.(6).

As the SNIR value for each user lies within a predefined range, then the user has only to feedback the AMC interval index (through the Channel Quality Indicator (CQI)), and not the SNIR value. Therefore, the number of required bits to feedback the SNIR value is reduced, and resources efficiency is increased. Notice that this is a modified feedback strategy as compared to the original

\footnotetext{
1 Even the beams (b) are orthogonal but their flows in the channel (hb) are not orthogonal [11].
} 


\begin{tabular}{|l|l|l|l|}
\hline CQI index & AMC & Efficiency $\zeta(\mathrm{bps} / \mathrm{Hz})$ & SNIR (dB) \\
\hline 0 & No service & 0 & $<-6.7$ \\
1 & QPSK, 1/4 & 0.55 & -6.7 \\
2 & QPSK, 1/3 & 0.835 & -4.7 \\
3 & QPSK, 1/2 & 1.12 & -2.3 \\
4 & QPSK, 2/3 & 1.35 & 0.2 \\
5 & QPSK, 3/4 & 1.69 & 2.4 \\
6 & 16-QAM, 1/4 & 1.98 & 4.3 \\
7 & 16-QAM, 1/3 & 2.56 & 5.9 \\
8 & 16-QAM, 1/2 & 3.045 & 8.1 \\
9 & 16-QAM, 2/3 & 3.43 & 10.3 \\
10 & 16-QAM, 3/4 & 3.815 & 11.7 \\
11 & 64-QAM, 1/4 & 4.2 & 14.1 \\
12 & 64-QAM, 1/3 & 4.585 & 16.3 \\
13 & 64-QAM, 1/2 & 4.97 & 18.7 \\
14 & 64-QAM, 2/3 & 5.355 & 21 \\
15 & 64-QAM, 3/4 & 5.75 & 22.7 \\
\hline
\end{tabular}

Table I: AMC intervals in LTE-A

MOB setup in section II-A, but it is the only way to adapt the feedback to the LTE-A standard.

We target an advanced AMC strategy where the objective is to achieve energy saving in the transmission process. The principle behind our strategy is that the predefined SER threshold will be satisfied whatever is the SNIR value within the AMC interval. Based on Table I, if a user $A$ measures an SNIR value of $6 \mathrm{~dB}$ and user $B$ measures an SNIR of $8 \mathrm{~dB}$ both will feedback the CQI index 7, and the eNB will serve them with the 16QAM modulation in order to satisfy their SER threshold. The eNB does not realize that the performance of users $A$ and $B$ is different as it is only informed about their CQI index.

Nevertheless, user $B$ shows much better channel characteristics (higher SNIR) that can be employed by the eNB to achieve energy saving. The SNIR difference of $2 \mathrm{~dB}$ between $B$ and $A$ can be exploited, so that the transmitted energy/power can be reduced to $B$, thus decreasing its measured SNIR value to the threshold value (i.e., same modulation is still used). Therefore, energy saving is obtained without causing any degradation to the system performance, as the same AMC interval is employed and the quality reception minimum threshold is satisfied. In order to formulate the saved energy $\left(E_{s}\right)$ we first define the amount of saved power $\left(P_{s}\right)$ as the difference between the total transmit power constraint $P_{t}$ (presented in the system model section) and the amount of required power within any interval as [13]

$$
P_{s}=P_{t}\left(1-\frac{\gamma_{r}}{\gamma}\right)
$$

where $\gamma_{r}$ is the predefined threshold SNIR value of any interval and $\gamma$ is the measured SNIR value.

Within the LTE-A standard only 4 bits are allocated to feedback the CQI, so that a maximum of 16 AMC intervals are possible (as shown in Table I). On the other hand, the LTE-A standard keeps 4 additional feedback bits for the MIMO transmission in TM-5. They are 1 bit for the Rank Indicator 
(RI) and 3 bits for the Precoding Matrix Indicator (PMI), that are always available in the TM-5 mode. The MOB scheme does not need for RI and PMI, thus we will employ these bits to indicate the SNIR value within each AMC interval. Generally speaking, when more information is available at the eNB about the channel status, better adaptation of the transmitted energy can be be accomplished, thus obtaining higher energy saving.

In order to give a general expression, we consider that there are $b$ extra bits for energy saving (if we employ the RI alone, then $b=1$ ). We propose that the UE subdivides each AMC interval into $G=2^{b}$ subregions with predefined thresholds, i.e., for the $r^{\text {th }}$ SNIR interval $\left[\gamma_{r}, \gamma_{r+1}\right)$ the thresholds can be set as $\left[\gamma_{r}, \gamma_{r}+\frac{L}{2^{b}}, \gamma_{r}+\frac{2 L}{2^{b}}, \ldots . ., \gamma_{r+1}\right]$, where $\mathrm{L}$ is the length of the SNIR interval, (i.e., $L=\gamma_{r+1}-\gamma_{r}$ ). If the users' measured SNIR value lies within the $g^{t h}$ subregion $\left[\gamma_{r}+\frac{L}{2^{b}}, \gamma_{r}+\frac{2 L}{2^{b}}\right)$, it will further feedback the index of that subregion. Now the eNB will assume that the SNIR value equals the lowest threshold of the $g^{\text {th }}$ subregion (i.e., $\gamma_{r}+\frac{L}{2^{b}}$ ), and it will reduce the transmitted energy to move the SNIR value $(\gamma)$ down from the $g^{\text {th }}$ subregion threshold to the threshold of the original SNIR interval $\left(\gamma_{r}\right)$, thus saving power/energy with the lowest possible number of bits.

The achieved power saving is the difference between the power needed for transmission when the standard approach is used, and when our scheme is employed, so that using Eqn.(8), the saved power per beam and for the $g^{t h}$ subregion we formulate it as

$$
P_{s}(g)=\frac{P_{t}}{n_{t}}\left(1-\frac{\gamma_{r}}{\gamma_{r}+\frac{L_{r} g}{2^{b}}}\right)
$$

where $g=\left[1,2, \ldots, 2^{b}-1\right]$ so that the saved energy per beam within the transmission interval $T_{F}$ and for the $g^{\text {th }}$ subregion we obtain it as

$$
E_{s}(g)=\int_{0}^{T_{F}} \frac{P_{t}}{n_{t}}\left(1-\frac{\gamma_{r}}{\gamma_{r}+\frac{L_{r} g}{2^{b}}}\right) d t=\frac{P_{t}}{n_{t}}\left(1-\frac{\gamma_{r}}{\gamma_{r}+\frac{L_{r} g}{2^{b}}}\right) T_{F}
$$

The average value of the saved energy from AMC deployment can be calculated by averaging the saved energy given in Eqn.(10) over the PDF of the SNIRs and within each one of the possible AMC intervals and over each subregion. We formulate the average saved energy per beam as

$$
\overline{E_{s}}=\sum_{r=0}^{R} \sum_{g=1}^{2^{b}-1} E_{s}(g) \int_{\gamma_{r}+\frac{g L_{r}}{2^{b}}}^{\gamma_{r}+\frac{(g+1) L_{r}}{2^{b}}} f_{\gamma}(\gamma) d \gamma
$$

where $L_{r}$ is the length of the $r^{t h}$ SNIR interval. Notice that within the interval $\left(\gamma<\gamma_{1}\right)$ the system is in outage and there is no transmission as the user cannot decode the signal if it is below the minimum detection value. Remind that within the MOB transmission strategy, there exists another source of energy saving when beams are switched off, as explained in section II. For our system 
where users are randomly distributed over the coverage area, the probability that there is a transmission on a given beam equals the probability that at least one user selects a given beam, which can be described as

$$
\Omega_{r_{\text {beam }}}=1-\left[1-\frac{1}{n_{t}}\right]^{K}
$$

Therefore the overall average saved energy $E_{t}$ over the $n_{t}$ beams must consider both situations, which enables us to formulate it as

$$
E_{t}=\left(\frac{P_{t}}{n_{t}} T_{F}\left(1-\Omega_{r_{\text {beam }}}\right)+\overline{E_{s}} \Omega_{r_{\text {beam }}}\right) n_{t}
$$

where $\left(P_{t} / n_{t}\right) T_{F}$ is the allocated energy per beam. Using the CDF for the SNIR given in Eqn.(6), the average value of the system saved energy we formulate it in a closed form expression as

$$
\begin{aligned}
E_{t}= & P_{t} T_{F}\left[1-\frac{1}{n_{t}}\right]^{K}+ \\
& +n_{t}\left(1-\left[1-\frac{1}{n_{t}}\right]^{K}\right) \sum_{r=0}^{R} \sum_{g=1}^{2^{b}-1} E_{s}(g)\left[F\left(\gamma_{r}+\frac{(g+1) L_{r}}{2^{b}}\right)-F\left(\gamma_{r}+\frac{g L_{r}}{2^{b}}\right)\right](14)
\end{aligned}
$$

where $K$ is the number of available users in the system. We should stress again that this is the achievable saved energy from our proposed scheme, but definitely, more energy can be reduced is we consider more subregions and/or we have more available CSIT. But all of such improvements would not be compliant with the LTE-A specs, which outstands the benefits of our proposal: to achieve energy decrease while being back-compatible.

\section{Multiuser MAC layer scheme}

In this section we will present an energy efficient MAC scheme for the proposed AMC strategy to account for the demands of the MOB transmission technique, and to support multiuser downlink transmission. We will show that the required modifications are back-compatible with the LTE-A standard. First, we briefly explain the LTE-A frame and later we will introduce the proposed modifications.

\subsection{LTE-A Frame}

We consider the Frequency Division Duplexing (FDD) mode for the downlink of an LTE-A system, where the total system bandwidth is divided into OFDM subcarriers, and the subcarriers are grouped into resource blocks which consists of 12 consecutive subcarriers in frequency domain [21]. The smallest resource 
unit that a user will be assigned is the Scheduling Block (SB). It consists of $Q$ consecutive RBs with a frame scheduling duration of $T_{F}$, and it is composed of multiple Transmission Time Intervals (TTIs), where each TTI is $1 \mathrm{~ms}$ duration and consists of 14 OFDM symbols [22].

We consider the semi-persistent scheduling at each downlink frame $T_{F}$ available for transmission at the physical layer. The first OFDM symbols are reserved as Physical Downlink Control Channel (PDCCH) for signaling the control information. It carries the scheduling information to the users such as information related to resource indication, the multi-antenna information, the modulation and coding scheme for the shared data, etc [23]. The remaining OFDM symbols are reserved as Physical Downlink Shared Channel (PDSCH) for data transmission, where one modulation and coding scheme is assigned to a user in each frame time interval, as its channel won't change during that time (i.e., less than the channel coherence time).

Before the data is transmitted within the $\mathrm{PDSCH}$, the data packets go through the link layer protocol, where each sub layer adds its header to the data units, forming transport blocks ready for transmission [1]. There are three sub layers in the design of an LTE-A link layer [24]: Packet Data Convergence Protocol (PDCP) which is responsible for the Internet Protocol (IP) header compression and ciphering, Radio Link Control (RLC) responsible of data segmentation and concatenation in addition to the Automatic Repeat reQuest (ARQ) process. The third layer is the MAC which supports scheduling operation of both Uplink and Downlink radio resources. Cyclic Redundancy Check (CRC) is attached to the data units to allow the receiver to detect bit errors and to forward error-free packets to the IP layer at the receiver.

\subsection{Multiuser MAC Protocol description}

The aim of the proposed scheme is to exploit the MU-MIMO channel capabilities, to opportunistically assign service to the user with the highest SNIR value per beam. These users will share the downlink resource blocks via the MOB transmission technique, thus not only maximizing the system throughput but also obtaining higher energy efficiency. This section explains how our proposal in section III fits within the LTE-A system, while being back-compatible.

In each frame scheduling time $\left(T_{F}\right)$ the eNB generates $n_{t}$ random beams and sends cell specific reference signals (RS) from each one of the $n_{t}$ beams. This is performed to support channel estimation for channel quality measurement of each of the $n_{t}$ beams [25]; enabling each user to calculate its received SNIR value for each one of the $n_{t}$ beams, to select the beam showing the largest SNIR value and to search the AMC interval where it falls. Then the corresponding CQI value plus an identifier for the beam index are fed back to the eNB. In order to allow the eNB to apply our proposed energy saving mechanism, each user sends additional $b$ bits to indicate the SNIR subregion within the CQI interval, as previously discussed in section III. 


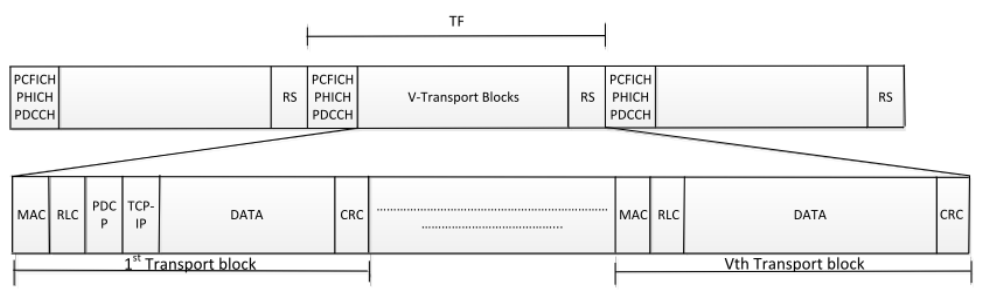

Fig. 1 LTE-A Frame structure.

Based on the CQI feedback, the eNB selects the user with the highest CQI on each beam, determines the appropriate modulation and coding scheme, applies energy saving mechanism based on the $b$ bit feedback, and then transmits simultaneously the data for the selected users with the calculated energies. Finally, each user receives its data and sends an Acknowledgement (ACK) indicator to certify the correct reception [1].

Notice that this technique can be implemented within LTE-A as it supports MU-MIMO in TM-5 with limited feedback, where the UE reports 1-bit RI, 3bits PMI together with 4-bits CQI [18], which add up to a total of 8 bits from each user. Our scheme requires to feedback $\left(4+\log _{2}\left(n_{t}\right)\right)$ bits from each user, 4 of them for the CQI and the $\left(\log _{2}\left(n_{t}\right)\right)$ as the beam index (i.e., when the eNB is equipped with 2 transmit antennas the UE has to feedback 1-bit, 2bits for 4 transmit antennas, and so on). Any number of antennas at the eNB can be considered within our proposal, but MIMO commercial deployment results have shown that the best results are obtained by using only a subset of 2 antennas to provide service to 2 users at each time, code and frequency block, and any increase in the number of serviced users shows negligible benefit as the multiplexing gain will not compensate the problem of interference and channel correlations. Therefore we will consider that two beams are generated, where our scheme saves 3 bits which can be used for energy saving. Obviously, increasing the number of considered beams would decrease the amount of saved feedback bits and energy.

The proposed LTE-A frame is shown in Fig. (1), where the first OFDM symbols are reserved for control signaling within each frame duration, while the data packets are organized into $V$ transport blocks available for transmission. Notice that if the length of the data to be transmitted is larger than the maximum transport block length, segmentation is done at the RLC layer [23]. Reference signals (RS) are sent over each TTI within the frame from the $n_{t}$ beams to keep the compatibility with the standard [25]. We assume that whenever the SNIR is above the minimum threshold, the receiver is able to correctly detect the transmitted signal (i.e., without errors), so that the ARQ retransmissions are not considered within the analysis nor simulations. 
4.3 System Data Rate

Our proposal is related to energy saving, but it also achieves large data rate as it provides simultaneous service to more than one user. As our proposal is based on AMC, then the achievable data rate is affected by the instantaneous reported SNIR values and their allocation to the different AMC intervals. Using the formulated CDF in section II, the achievable sum data rate $\Upsilon$ we calculate it as

$$
\Upsilon=B_{w} n_{t}\left(1-\left[1-\frac{1}{n_{t}}\right]^{K}\right) \sum_{r=1}^{R} \zeta(r)\left[F_{\gamma}\left(\gamma_{r+1}\right)-F_{\gamma}\left(\gamma_{r}\right)\right]
$$

where $\zeta(r)$ is the spectral efficiency of bps $/ \mathrm{Hz}$ and for each one of the AMC intervals from Table I; while $B_{w}$ is the available bandwidth. Using Eqn.(15) and Eqn.(6), we represent the average system sum rate with all the involved parameters as

$$
\Upsilon=B_{w} n_{t}\left(1-\left[1-\frac{1}{n_{t}}\right]^{K}\right) \sum_{r=1}^{R} \zeta(r)\left\{\left[1-\frac{e^{-n_{t} \gamma_{r+1} \sigma^{2}}}{\left(1+\gamma_{r+1}\right)^{n_{t}-1}}\right]^{K}-\left[1-\frac{e^{-n_{t} \gamma_{r} \sigma^{2}}}{\left(1+\gamma_{r}\right)^{n_{t}-1}}\right]^{K}\right\}
$$

\subsection{System Throughput}

In order to make a fair comparison in terms of the data rate of MOB to other schemes in the literature and/or the standard, the feedback load must be included in the data rate formulation. An interesting metric is the throughput $(\Psi)$ that accounts for the effective rate at which the data can be sent through a communication channel, taking into consideration the control signaling and feedback overhead. Remind that MOB scheme is based on the availability of several users and on selecting the one with the largest SNIR value for each beam, thus increasing the number of available users would increase MOB performance but at expenses of larger feedback load in the system. Beside the improvement in data rate and energy saving, the operator is also concerned about the consumed resources to achieve them.

We formulate a metric that jointly accounts for the control signaling (i.e., $\mathrm{PDCCH}, \mathrm{RS}$, etc), the header within the PDSCH (i.e., MAC, RLC, etc), and the feedback as CQI, PMI and ACK in the performance evaluation. Even the feedback load is sent via the PUCCH [25] and not on the same downlink shared channel, but it should be included in the performance evaluation.

As the proposed downlink frame is organized into $Q \mathrm{RBs}$ in frequency domain and a scheduling period of $T_{F}$, then a total number of resource elements $\left(R E_{t o t}=12 \cdot Q \cdot 14 \cdot T_{F}\right)$ exists within each frame, where a resource element can be defined as one allocated subcarrier in one OFDM symbol. As we stated earlier a number of the available REs are reserved for the downlink control signaling $\left(R E_{D L C}\right)$; while $\left(R E_{U L C}\right)$ are composed of the bits employed for 
the feedback in the physical channel PUCCH and it has also to be included in our calculations, making the percentage of REs available for data transmission $R E_{\text {data }}$ to be formulated as

$$
R E_{\text {data }}=\frac{\left(R E_{t o t}-R E_{D L C}\right)}{\left(R E_{t o t}+R E_{U L C}\right)}
$$

where $R E_{U L C}$ is sent each frame duration $\left(T_{F}\right)$. To mathematically formulate $R E_{U L C}$ notice that it consists of CQI bits $\left(b_{C Q I}\right)$, PMI bits $\left(b_{P M I}\right)$ and ACK bits $\left(b_{A C K}\right)$ all of them modulated into QPSK complex symbols (i.e., 2 bits per symbol) before mapping them into REs [23]. Then we obtain $R E_{U L C}$ as

$$
R E_{U L C}=\frac{K \cdot b_{C Q I}+K \cdot b_{P M I}+n_{t} \cdot b_{A C K}}{2}
$$

where $K$ is the number of users in the system and $n_{t}$ selected users at the same frame. It remains to obtain the expression for the number $B$ of bits sent within each frame through the PDSCH, that using the previous expression we express it as

$$
B=\Upsilon \frac{\left(R E_{t o t}-R E_{D L C}\right)}{R E_{t o t}} T_{F}
$$

where $\Upsilon$ is given in Eqn.(16). $B_{\text {head }}$ bits out of $B$ are employed as header (i.e., MAC, RLC, etc) and looking to Fig. (1), we formulate it as

$$
B_{\text {head }}=T C P_{\text {header }}+P D C P_{\text {header }}+V\left(R L C_{\text {header }}+M A C_{\text {header }}+C R C\right)
$$

With all above expressions, we can state the throughput expression as

$$
\Psi=\Upsilon\left(1-\frac{B_{\text {head }}}{B}\right) R E_{\text {data }}
$$

\section{Simulation Results}

In order to asses the behavior of the proposed scheme within LTE-A, its performance is evaluated by Monte Carlo simulations. A multiuser MIMO scenario is considered where the eNB transmitter is equipped with any number of antennas, but only 2 beams are generated and a total number of $K=15$ users are available in the cell. All the users are assumed to have the same average channel characteristics, and showing the same distribution for the maximum SNR value, so that each user has the same probability to be selected. If this is not the case (e.g. heterogeneous users distribution in the cell, with some users far from the eNB), then a channel normalization (e.g. division by the path loss) can be accomplished for such a scenario.

Within the semi-persistent scheduling, the transmitted data in terms of transport blocks are organized into frames of $T_{F}=20 \mathrm{~ms}$ duration, each user will be assigned the whole resource blocks within the $20 \mathrm{~ms}$ interval, so that 


\begin{tabular}{|l|l|l|}
\hline Parameter & Single-user & Two-users \\
\hline Bandwidth & $5 \mathrm{MHz}$ & $5 \mathrm{MHz}$ \\
Number of subcarriers & 300 & 300 \\
Number of (RBs) & 25 & 25 \\
Frame duration & $20 \mathrm{~ms}$ & $20 \mathrm{~ms}$ \\
Transmitted power & $1 \mathrm{~W}$ & $1 \mathrm{~W}$ \\
Max.size of transport block & 6144 & 6144 \\
Control signaling overhead & $9.15 \%$ & $25.37 \%$ \\
TCP-IP header & 8 bytes & 16 bytes \\
PDCP header & 4 bytes & 8 bytes \\
RLC header & 2 bytes & 4 bytes \\
MAC header & 2 bytes & 4 bytes \\
CRC & 3 bytes & 6 bytes \\
CQI & 4 bits / 20ms & 8 bits / 20ms \\
PMI-beam index & - & 1 bit / 20ms / user \\
PMI-energy saving & - bit / 20ms / user \\
ACK & 1 bit / 4ms & 1 bit / $4 \mathrm{~ms} \mathrm{/} \mathrm{user}$ \\
\hline
\end{tabular}

Table II: LTE-A Simulation parameters.

through MOB, a maximum of 2 users can be served during that time. The control information (scheduling information) for all the selected users are transmitted via PDCCH within the first OFDM symbol. The data headers such as PDCP, RLC and MAC for all the selected users are transmitted within each transport block on the PDSCH. It is assumed that the system is saturated, so that there is always available packets for all the associated users. The simulation parameters are summarized in Table II.

We will compare three different schemes. The first one is the benchmark single serviced user scheme, where one beam is generated at the transmitter to serve only one randomly selected user, so that after the eNB randomly selects a user for transmission, that user will feedback a CQI based on its measured Signal to Noise Ratio (SNR) value to allow the eNB to apply AMC; this strategy is the lowest feedback requirement one. The second scheme is the opportunistic single user service, where only one beam is generated and each user in the system feeds back its CQI then the eNB selects the best user. Finally the third one is our proposed scheme where the eNB generates two orthogonal beams, each user feeds back a CQI and PMI (corresponding to the selected beam and to allow applying energy saving at the eNB), then the eNB selects the best user for each one of the beams. No other LTE-A compliant schemes are found in the literature that provide energy saving, so that our benchmark scheme is fixed to be the one randomly selected users without energy saving objectives.

The throughput performance of the three schemes is shown in Fig. (2) for a scenario with a variable number of users. We consider that the compared schemes use the same energy restriction for a fair comparison in performance. The figure illustrates that our proposal outperforms the opportunistic single user scenario (i.e., scheme 2) for realistic number of users. Although our scheme induces a feedback load that enlarges as the number of users increases together with control signaling, but it is obvious that also greater diversity and multiplexing gain are obtained, which translates into a larger system throughput. Notice that the figure shows very good matching between the simulations and the mathematically obtained results. 


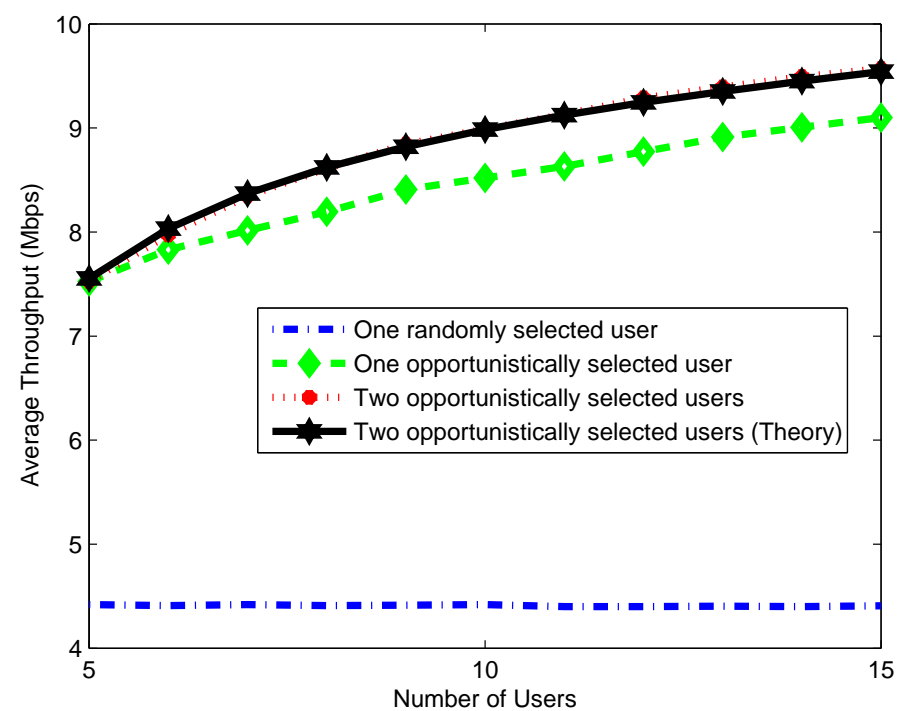

Fig. 2 Throughput comparison for the different schemes within an LTE-A scenario.

Significant energy saving is achieved with the lowest possible number of feedback bits and keeping the backward compatibility with the standard. To further improve the energy saving, a user may be allowed to feedback 2 bits instead of 1 bit, by dividing each AMC interval into 4 sub regions and applying the same procedure stated in section III. Obviously, a larger feedback load is required, but still being backward compatible with the LTE-A Standard.

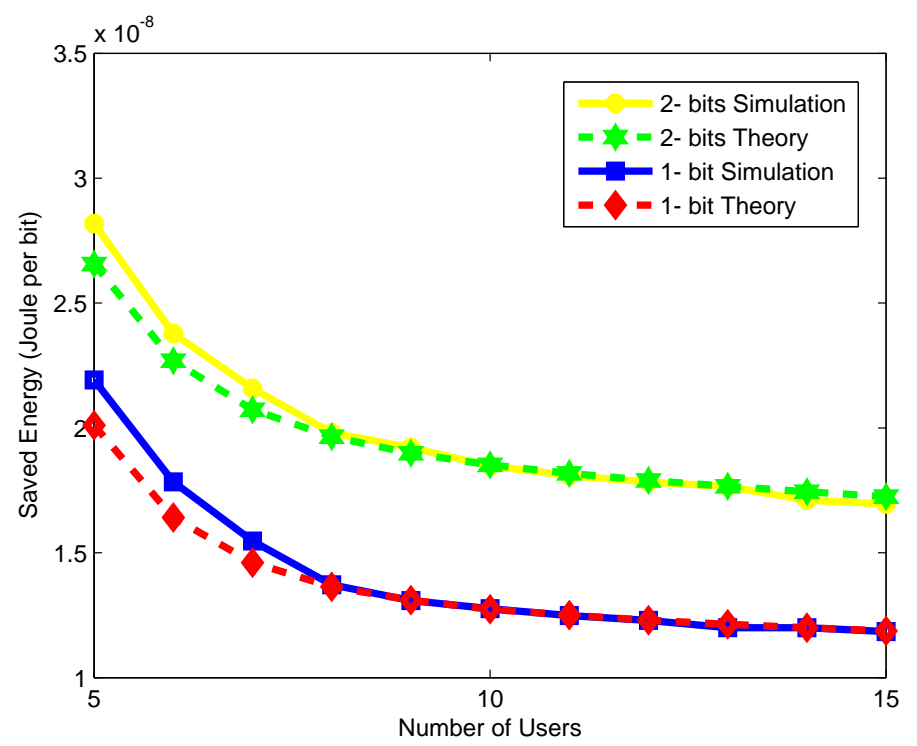

Fig. 3 The saved energy within our scheme for a variable number of users in the cell. 


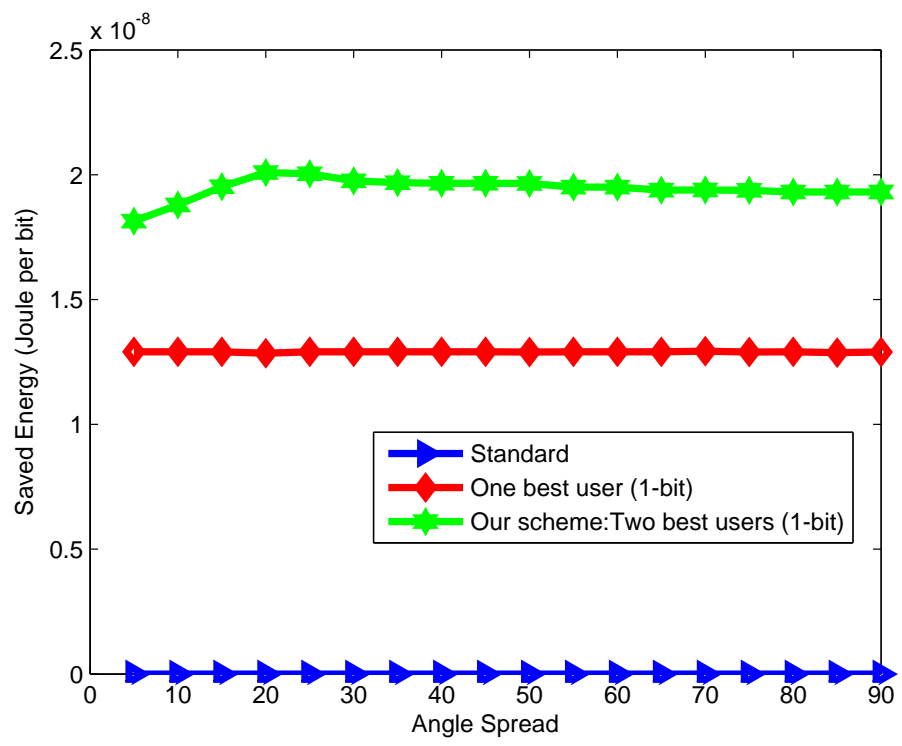

Fig. 4 The saved energy within our scheme for different angle spread values

The resultant saved energy within our scheme is plotted in Fig. (3) for the cases of 1 bit and 2 bits feedback. Bear in mind that the current release of LTE-A does not provide any MAC energy saving design, therefore, we can consider the benchmark design showing no energy saving in Fig. (3). Notice that with low number of users there exists a higher probability that an eNB only finds one suitable user above the minimum detection threshold, so that the other beam is switched off and its energy is saved, as explained in section II-A. We obtain that 2 bits feedback improves the performance of energy saving by almost $30 \%$. Fig. (3) also plots the results from the mathematically obtained results in Eqn. (14) and how they show a very good matching with the simulations.

Finally, to present the performance of our proposed scheme within different environments where 1 bit feedback for energy saving is employed. For small AS values the channel is very directional (i.e., strong Rician component), so that the transmission is without a lot of interference, but also the probability that a randomly generated beam matches with the channel is lower. The two effects almost cancel each other as can be seen in Fig. (4), that displays the saved energy for different values of angle spread when there are 7 users in the system. It shows that our scheme behaves better for all kinds of AS (i.e., covering both indoor and outdoor channels).

\section{Conclusions}

In this paper we discuss a new energy efficient MAC scheme that supports MUMIMO transmission, which is combined with $\mathrm{MOB}$ and $\mathrm{AMC}$ at the physical 
layer. To exploit the capabilities of the MU-MIMO scenario, opportunistic selection of the best set of users for transmission is accomplished. The simulation results show that although our scheme requires feedback payload at the MAC layer to simultaneously serve several users, it shows an improved performance compared to single user techniques. Our proposal exploits AMC to apply energy saving mechanism at the transmitter without causing any degradation in the system performance, while being back-compatible with the LTE-A specs. We formulate both the energy saving and throughput of our proposal through closed form expressions that match the computer-based simulations.

\section{References}

1. A. Larmo et al., "The LTE link-layer design," IEEE Communications Magazine, vol. 47, no. 4, pp. 52 - 59, April 2009.

2. L. Zhang et al., "Quality of Service Modelling of Virtualized Wireless Networks: A Network Calculus Approach", Springer Mobile Networks and Applications, Vol.19, no.4, August 2014

3. D. Astely et al., "LTE: the evolution of mobile broadband," IEEE Communications Magazine, vol. 47, no.4, pp. 44 - 51, April 2009.

4. H. Ekstrom et al., "Technical solutions for the 3G long-term evolution," IEEE Communications Magazine, vol. 44, no. 3, pp. 38 - 45, March 2006.

5. M. Mustaqim et al., "LTE-Advanced: Requirements and Technical Challenges for 4G Cellular Network," CIS Journal, vol. 3, no. 5, May 2012.

6. H. Zhang et al., "MIMO downlink scheduling in LTE systems," IEEE INFOCOM 2012, pp. 2936 - 2940, March 2012

7. Q. Li et al., "MIMO Techniques in WiMAX and LTE: A Feature Overview," IEEE Communications Magazine, vol. 48, no. 5, pp. 86 - 92, May 2010.

8. R. Kwan and C. Leung, "A survey of scheduling and interference mitigation in LTE," Journal of Electrical and Computer Engineering, vol. 2010, no.1, January 2010.

9. T. Samarasinghe et al., "The Feedback-Capacity Tradeoff for Opportunistic Beamforming," IEEE ICC, Kyoto, June 2011.

10. M. Sharif and B. Hassibi, "On the Capacity of MIMO Broadcast Channel with Partial Side Information," IEEE Trans. Inform. Theory, vol. 51, no. 2, February 2005.

11. N. Zorba and A.I. Perez-Neira, "CAC for Multibeam Opportunistic Schemes in Heterogeneous WiMax Systems Under QoS Constraints," IEEE Globecom, New Orleans, November 2007.

12. W. Vereecken et al., "Power consumption in telecommunication networks: overview and reduction strategies," IEEE Communications Magazine,vol. 49, no.6, pp. 62-69, June 2011.

13. S. Althunibat et al., "Energy optimization in multiuser quantized feedback systems", EURASIP Journal on Wireless Communications and Networking, vol.83, August 2013.

14. R. Xie, F.R. Yu and H. Ji, "Interference Management and Power Allocation for EnergyEfficient Cognitive Femtocell Networks", Springer Mobile Networks and Applications, vol.18, no.4, August 2013.

15. A. Bousia et al., Green Distance-Aware Base Station Sleeping Algorithm in LTEAdvanced, IEEE ICC, Ottawa, June 2012.

16. X. Zhang et al., "Energy-Efficiency Study for Two-tier Heterogeneous Networks (HetNet) Under Coverage Performance Constraints" Springer Mobile Networks and Applications, vol.18, no.4, August 2013.

17. J. Huang et al., "A close examination of performance and power characteristics of $4 \mathrm{G}$ LTE networks," Proceeding MobiSys '12, pp. 225-238, New York, 2012

18. 3GPP TS 36.213, "Evolved Universal Terrestrial Radio Access (E-UTRA) Physical layer procedures," Rel. 12, March 2014. 
19. D. Gesbert et al., "Transmit Correlation-aided Scheduling in Multiuser MIMO Networks," IEEE ICASSP, May 2006.

20. P. Viswanath et al., "Opportunistic beamforming using dumb antennas," IEEE Trans. Inform. Theory, vol.48, pp. 1277 - 1294, 2002.

21. S. Saha and R.Quazi, "Priority-coupling-a semi-persistent MAC scheduling scheme for VoIP traffic on 3G LTE," 10th International Conference on Telecommunications, pp. 325 - 329, June 2009.

22. F. Shamshad et al., "Physical Layer Aspects of 3GPP's Long Term Evolution (LTE)," Advances in Computer Science and its Applications (ACSA),vol. 2, no.1, 2012

23. 3GPP TS 36.212, "Evolved Universal Terrestrial Radio Access (E-UTRA); Multiplexing and channel coding," Rel. 12, July 2014.

24. T. Tran et al., "Overview of enabling technologies for 3GPP LTE-advanced," EURASIP Journal on Wireless Communications and Networking, vol. 2012, no. 1, February 2012.

25. 3GPP TS 36.211, "Evolved Universal Terrestrial Radio Access (E-UTRA) Radio Physical Channels and Modulation," Rel. 12. March 2014. 